\title{
The Role of Irinotecan-Bevacizumab as Rescue Regimen in Children With Low-Grade Gliomas: A Retrospective Nationwide Study in 72 Patients
}

Charles de Marcellus ( $\nabla$ charles.de-marcellus@aphp.fr)

Institute Gustave-Roussy: Gustave Roussy https://orcid.org/0000-0003-3935-0924

Arnault Tauziède-Espariat

Hôpital Sainte-Anne: Hopital Sainte-Anne

Aurélie Cuinet

Gustave Roussy

Claudia Pasqualini

Gustave Roussy Institute: Gustave Roussy

Matthieu P. Robert

Hôpital Necker-Enfants Malades: Hopital universitaire Necker-Enfants malades

Kévin Beccaria

Hôpital Necker-Enfants Malades: Hopital universitaire Necker-Enfants malades

Stéphanie Puget

Hopital Necker-Enfants Malades: Hopital universitaire Necker-Enfants malades

Nathalie Boddaert

Hopital Necker-Enfants Malades: Hopital universitaire Necker-Enfants malades

Dominique Figarella-Branger

CHU Timone: Hopital de la Timone

Emilie De Carli

CHU Angers: Centre Hospitalier Universitaire d'Angers

Franck Bourdeaut

Institute Curie: Institut Curie

Pierre Leblond

Oscar Lambret Cancer Centre: Centre Oscar Lambret

Fanny Fouyssac

CHU Nancy: Centre hospitalier regional universitaire de Nancy

Nicolas Andre

APHM: Assistance Publique Hopitaux de Marseille

Anne I. Bertozzi

CHU Toulouse: Centre Hospitalier Universitaire de Toulouse

Thibault Butel 
Gustave Roussy Institute: Gustave Roussy

\section{Christelle Dufour}

Gustave Roussy Institute: Gustave Roussy

Dominique Valteau-Couanet

Gustave Roussy Institute: Gustave Roussy

\section{Pascale Varlet}

Hôpital Sainte-Anne: Hopital Sainte-Anne

Jacques Grill

Gustave Roussy Institute: Gustave Roussy

\section{Research Article}

Keywords: bevacizumab, irinotecan, children, low grade glioma

Posted Date: November 11th, 2021

DOl: https://doi.org/10.21203/rs.3.rs-1034556/v1

License: (c) (i) This work is licensed under a Creative Commons Attribution 4.0 International License. Read Full License

Version of Record: A version of this preprint was published at Journal of Neuro-Oncology on March 3rd, 2022. See the published version at https://doi.org/10.1007/s11060-022-03970-4. 


\section{Abstract}

\section{Introduction.}

At least half of children with low-grade glioma (LGG) treated with first line chemotherapy experience a relapse/progression and may therefore need a second-line chemotherapy. Irinotecan-bevacizumab has been recommended in this setting in France after encouraging results of pilot studies. We performed a retrospective analysis to define the efficacy, toxicity and predictors for response to the combination on a larger cohort.

\section{Methods.}

We reviewed the files from children $<19$ years of age with progressive or refractory LGG treated between 2009 and 2016 in 7 French centers with this combination.

\section{Results.}

72 patients (median age 7.8 years [range, 1-19]) received a median of 16 courses (range, 3-30). The median duration of treatment was 9 months (range, 1.4-16.2). $96 \%$ of patients experienced at least disease stabilization. The 6-month and 2-year progression-free survivals (PFS) were $91.7 \%$ [IC 95\% 85.598.3] and 38.2\% [IC 95\% 28.2-51.8] respectively. No progression occurred after treatment in 18 patients with a median follow-up of 35.6 months (range, 7.6-75.9 months). Younger patients had a worse PFS $(p=0.005)$. Prior chemoresistance, NF1 status, duration of treatment, histopathology or radiologic response did not predict response. The most frequent toxicities related to bevacizumab included grades 1-2 proteinuria in 21, epistaxis in 10, fatigue in 12 and hypertension in 8 while gastro-intestinal toxicity was the most frequent side effect related to irinotecan.

\section{Conclusion.}

Bevacizumab-irinotecan is highly effective for children with recurrent LGG who have failed standard chemotherapy regimens whatever their clinical characteristics, only younger children had a worse PFS.

\section{Introduction}

Low-grade gliomas (LGG) are the most common form of childhood brain tumor, accounting for one third of all primary central nervous system tumors in children less than 18 years of age [1]. Prognosis is excellent when complete resection is obtained [2]. However, LGG are frequently not amenable to a complete resection because of their location. In patients with incompletely resected tumors, further treatment is required in up to $50 \%$ of patients at two years [3]. Patients with recurrent LGG usually receive chemotherapy or focal radiotherapy, albeit less frequently due to the risk of long-term toxicity [4]. 
Despite important response rates, most children relapse within the first three years following first-line chemotherapy completion [5-10]. Indeed, most children with optic pathway glioma treated in the BBSFOP study that experienced a first relapse subsequently recurred again and up to $25 \%$ of the LGG recurring after the first line chemotherapy ultimately died from the disease [11]. New therapeutic strategies have therefore to be developed in order to improve the poor outcome of refractory or recurrent LGG.

Besides conventional chemotherapy, anti-angiogenic drugs have been proposed for recurrent LGG. Indeed, neoangiogenesis has been associated with more aggressive forms of LGG [12]. Vascular endothelial growth factor (VEGF) is the best characterized pro-angiogenic factor associated with tumor growth [13]. Bevacizumab is a recombinant humanized monoclonal immunoglobulin (IgG1) antibody and has a direct anti-angiogenic effect by binding to and clearing VEGF from the tumor environment; this drug may have a direct role on vascular normalization, improving delivery of cytotoxic drugs. Inhibiting VEGF seems to normalize the vasculature and enable a better blood supply to be transiently established, producing a window where the access and efficacy of chemotherapy will be increased [14]. Moreover, it decreases interstitial pressure and hereby the injury induced by the tumor infiltration [15]. Irinotecan is a campothecin derivate that inhibits the nuclear enzyme, topoisomerase I. It penetrates the blood-brain barrier and has shown single agent anti-tumor efficacy against recurrent glioma $[16,17]$. It may as well potentiate the action of bevacizumab by inhibiting hypoxia-inducible factor 1 [18].

The strategy of inhibiting angiogenesis by blocking the pathway of the VEGF has proved successful in several adult solid tumors, when combined with systemic cytotoxic treatment, opening new therapeutic perspectives for children as well [19-24]. Only few small pediatric series combining these two drugs have been reported so far but with encouraging results and mechanistic studies suggest a crucial role for VEGF receptor-induced angiogenesis in the progression and maintenance of pediatric pilocytic astrocytoma [25-28]. The initial case series evaluating this treatment approach has demonstrated objective responses and clinical improvements in seven of the ten children treated with recurrent LGG, and no tumor progression while on treatment [25]. These results have been confirmed by the same authors with an expanded group of 35 pediatric patients in a multi-institutional phase II trial conducted by the Pediatric Brain Tumor Consortium [29]. The treatment was thus recommended by the brain tumor committee of the Societe Française des Cancers de l'Enfant (SFCE) as a rescue regimen in children with LGG.

The role of this combination in the management of LGG has to be further defined. We decided therefore to undertake a larger multicentric retrospective population-based study with longer follow-up to define the characteristics associated with sustained response to this therapy.

\section{Patients And Methods}

\section{Patients/ Eligibility Criteria}


This study involved children $<19$ years of age with progressive or refractory low-grade glioma treated between 2009 and 2016 in centers of the Societe Française des Cancers de l'Enfant (SFCE) with the association of bevacizumab and irinotecan. The study was approved by the IRB of Gustave Roussy and by the Comité Consultatif sur le Traitement de l'Information en matière de Recherche (CCTIRS $\mathrm{n}^{\circ}{ }^{14.817)}$. Accordingly, the parents/guardians gave their written permission for retrospective anonymized data collection and analysis.

Eligible histological diagnosis included all LGG. Histologic confirmation was not required for patients with optic/hypothalamic tumors, especially in case of neurofibromatosis type 1 (NF1). Whenever possible, the status of the BRAF gene (re-arrangement or mutation) was also determined by immunohistochemistry, sequencing or digital droplet PCR (ddPCR) for mutations and by FISH or ddPCR for duplications. The date of diagnosis was the date of the biopsy, or the date of the first magnetic resonance imaging (MRI) scan otherwise. In order to be eligible for the study patients had to have received at least one prior line of nonsurgical therapy. Refractory tumors were also eligible. At the time of treatment, all patients had evidence of clinical and/or radiographically progression and had radiographically evaluable disease.

\section{Treatment}

All patients were treated with bevacizumab and irinotecan, using the regimen previously published [29, 30]. Bevacizumab was given at $10 \mathrm{mg} / \mathrm{kg}$ and irinotecan at $125 \mathrm{mg} / \mathrm{m}^{2}$ every two weeks initially. Patients were treated with the drug association at the discretion of the physician as best available treatment. Patients reports were collected for review of clinical and radiological files. Patients characteristics, including age at start of bevacizumab, gender, histopathology, molecular biology of the tumor, number of prior treatment lines, duration of treatment, number of cycles administered, time to best clinical response, time to progression, were collected. Same data were also collected in patients rechallenged with bevacizumab. Side effects were collected and their assessment was based on the Common Terminology Criteria for adverse events $v 4.0$.

\section{Evaluation of responses/ Assessment of efficacy}

All patients underwent an MRI, with and without contrast, prior to treatment initiation and at 2-3 month intervals, thereafter. Response assessed by the treating institution based upon RANO criteria were collected [31]. Best response anytime were considered. Modifications of the contrast enhancement only were quoted but not considered as true responses. Clinical responses were also noted separately.

\section{Statistical Analysis}

The primary endpoint to evaluate prognostic factors was progression-free survival (PFS), defined as the interval from the first day of chemotherapy to the date of the first event (progression, relapse, second malignancy, or death) or to the date of the last follow-up visit. The secondary endpoints were overall survival (OS) and response rate. Survival curves were estimated using the Kaplan-Meier method. Discrete variables were compared using the Fisher exact test. Normally distributed continuous variables were 
compared using Student $t$ test, and non-normally distributed data were compared using the Wilcoxon rank-sum test. All analyses were performed with $\mathrm{R}$ version 3.3.1 (The R Core Team. R: 2016).

\section{Results}

\section{Patient Characteristics}

During the study period, 84 children with recurrent LGG were treated with this bevacizumab-based regimen but only 72 met the inclusion criteria. The 12 patients excluded had been treated with bevacizumab-irinotecan as first line of treatment. Patients characteristics are listed in Table 1. Median age at diagnosis was 3.2 years (range 2.5 months to 13 years) and median age at initial treatment was 7.8 years (range 1 to 19 years). Forty-seven patients (65\%) had an optic pathway glioma. Fifteen patients $(20.8 \%)$ had NF1. Thirteen patients (18\%) suffered from a multifocal disease, all of them together with an optic pathway tumor. Eight of them had NF1. Seven patients $(9.7 \%)$ presented with a leptomeningeal dissemination. Histopathology was obtained in fifty-nine patients (82\%) with a diagnosis of pilocytic astrocytoma for thirty-seven patients (Table 1). BRAF molecular status explorations found 28 KIAA1549:BRAF fusion (38.8\%), 6 BRAFV600E mutation (8.3\%), 12 BRAF wild type (16.6\%) and 15\% of undetermined status. Prior to this treatment, all patients had failed at least one prior chemotherapy and/or radiotherapy. The median number of prior lines of non-surgical treatment was 2 (range 0 to 6 lines). Forty-one tumors (57\%) had received prior surgical resection. 
Table 1

Clinical characteristics in 72 patients treated with irinotecan and bevacizumab.

\begin{tabular}{|ll|}
\hline Clinical characteristics & No. of patients \\
\hline Total number of evaluable patients & 72 \\
\hline Median age at initial treatment (range) & 7.8 years $(1-19)$ \\
\hline Histopathology & $43(60 \%)$ \\
\hline Pilocytic/ Pilomyxoid astrocytoma & $8(11 \%)$ \\
\hline Ganglioglioma & $21(29 \%)$ \\
\hline Optic pathway glioma (not biopsied) & $15(20.8 \%)$ \\
\hline No. of patients with NF1 & $13(18 \%)$ \\
\hline No. of patients with multifocal tumor & $7(9.7 \%)$ \\
\hline No. of patients with metastatic disease & $2(0-6)$ \\
\hline Median number of prior lines of chemotherapy (range) & \\
\hline Prior therapy & $70(97 \%)$ \\
\hline Chemotherapy & $1(1.4 \%)$ \\
\hline Radiotherapy & $1(1.4 \%)$ \\
\hline Chemotherapy + Radiotherapy &
\end{tabular}

\section{Responses and progression-free survival}

The median duration of treatment was 9 months (range, 1.4-16.2 months) with a median number of doses of bevacizumab received of 16 (range, 3-30 doses). The median follow-up from the first dose of irinotecan and bevacizumab was 43.6 months (range, 7.6-90 months). Objective responses defined as a radiological partial response, were noted in only 15 patients (21\%). Minor responses were observed in 16 patients (22\%) and stable diseases in 37 patients (51.5\%). $82 \%$ of patients showed a decrease in contrast uptake. Four patients suffered progressive disease on treatment at a median of 3.3 months from the start of therapy (range, 1.5-5.8). Best responses were noted at a median time of 10.5 weeks (range, 4-48 weeks) and at a median number of courses of chemotherapy of 6 (range, 2-21). Treatment and outcomes are represented in Figure 1.

Clinical improvement was noted in 35 patients (49\%). Of the 47 patients with an optic pathway glioma, vision improved in 21 (45\%) and remained stable in 26 (55\%). None of them had worsening of vision under chemotherapy. Of the 25 patients with LGG located elsewhere than in the optic pathway, 14 (56\%) described a neurological improvement such as neurodevelopmental gains, improved hemiparesis and/or 
better pain control and eleven (44\%) a stabilization of the symptoms. No patient had symptoms worsening.

Three-year overall survival and PFS for the entire population were $89.4 \%$ [IC 95\% 82.3-97.2] and 25\% [IC 95\% 16.2-38.3], respectively and the median PFS was 17.6 months (range, 2.3-75.9) with 25 patients (35\%) presenting a PFS $>24$ months. (Figure 2) The median PFS of the 54 patients who progressed was 16 months. No progression occurred in 18 patients with a median follow-up of 35.6 months (range, 7.675.9 months).

\section{Prognostic factors for response to therapy}

The study of risk factors as a function of radiological response (PR+MR vs SD+PD) showed that age at treatment initiation between 5 and 10 years was statistically associated with radiological response (OR $5.78[1.3-37.5] p=0.03)$. Treatment duration longer than 9 months and the number of irinotecanbevacizumab doses received ( $>15$ doses) were also associated with an improved radiological response (respectively, OR $4.6[1.4-16.4] p=0.005$ and OR 6.2 [1.8 - 26.0] $p=0.002$ ). Previous chemosensitivity, NF1 status or the status of the BRAF gene were not associated with the radiological response.

Age was associated with PFS; patients younger than 5 had a worse PFS than those older than 10 years, while those aged between 5 and 10 years old had an intermediate PFS, $p=0.005$ (figure 3 ). The analysis of the PFS according to the treatment duration demonstrated that most patients with PFS $>18$ months were treated more than 9 months $(67 \%)$. The median PFS of patients treated more than 9 months was higher than those treated for a shorter time: 19.6 months (range, 11-50 months) vs 15.8 months (range, 4.6-75.8 months). This difference, however, was not statistically significant, $(p=0.145)$. Time off treatment and before progression, i.e. PFS after stopping therapy, was the same in the two groups: 9.1 months in patients treated $<9$ months and 9.3 months in patients treated $>9$ months. Progression during therapy and patients treated recently and still on therapy were excluded from this analysis. Outcomes of these analyses are presented in Table 2 and supplementary data figure 1 to 7. 
Table 2

Risk factor analyses for PFS

\begin{tabular}{|c|c|c|}
\hline Clinical factors & Median PFS (range) & $p$ value \\
\hline Age at initial treatment & & 0.005 \\
\hline$<5$ years & 14.4 months (3-33.4) & \\
\hline $5-10$ years & 19 months $(5.1-54.1)$ & \\
\hline$>10$ years & 24.7 months (2.3-75.9) & \\
\hline No. of prior lines & & 0.74 \\
\hline 1 line & 16.2 months $(2.3-54.1)$ & \\
\hline$>1$ line & 17.4 months $(3-75.9)$ & \\
\hline Treatment duration & & 0.22 \\
\hline$<9$ months & 15.8 months (4.6-75.9) & \\
\hline$>9$ months & 19.6 months (11.1-53.9) & \\
\hline Bevacizumab doses & & 0.69 \\
\hline$<15$ doses & 13.5 months (4.6-75.9) & \\
\hline$>15$ doses & 19.6 months $(9.7-54.1)$ & \\
\hline Chemosensibility & & 0.84 \\
\hline Chemosensitive & 15.8 months $(5.1-39.4)$ & \\
\hline Chemoresistant & 18.8 months $(2.3-75.9)$ & \\
\hline NF1 & & 0.9 \\
\hline Yes & 19.3 months (4.63-54.1) & \\
\hline No & 18.3 months (2.3-75.9) & \\
\hline Radiological response & & 0.9 \\
\hline SD (stable disease) & 18.1 months (2.3-54.1) & \\
\hline MR/PR (minor/partial response) & 19.3 months (5.1-75.9) & \\
\hline Histopathology & & 0.22 \\
\hline Pilocytic astrocytoma KIAA1549:BRAF & 18.8 months $(2.3-75.9)$ & \\
\hline Ganglioglioma & 13.7 months $(6.1-33.4)$ & \\
\hline Others LGG & 14.9 months (3-44.8) & \\
\hline BRAF status & & 0.25 \\
\hline
\end{tabular}




\begin{tabular}{|ll|}
\hline Clinical factors & Median PFS (range) $\quad$ p value \\
\hline BRAF fusion & 18.8 months (2.3-39.4) \\
\hline BRAFV600E & 13.7 months (6.1-75.9) \\
\hline BRAF Wild Type & 15 months (3-44.8) \\
\hline Undetermined & 31 months (6.2-53.9) \\
\hline
\end{tabular}

\section{Toxicity}

The common grade 1-3 toxicities related to bevacizumab and irinotecan are listed in Table 3 (Supplementary data). The most common toxicities related to bevacizumab were grade 1-3 proteinuria, grades 1-2 epistaxis and hypertension in 22, 10 and 8 patients, respectively. The most common adverse events in initial treatment courses related to irinotecan were gastrointestinal toxicity. Gastrointestinal toxicity resolved when irinotecan was discontinued $(n=6)$. Treatment was discontinued in fifteen patients for the previously mentioned toxicity: proteinuria (7), gastrointestinal toxicity (6) and fatigue (2). Proteinuria resolved over a period of 2 weeks to 9 months following cessation of bevacizumab. Treatment was never discontinued because of hypertension. One patient came off treatment because of a grade 2 thrombosis of a deep vein after five months. The thrombosis did recur when the patient was retreated by bevacizumab and irinotecan for a clinical progression. No child in the study developed intracerebral hemorrhage. There was no toxic death.

Table 3: Toxicity due to bevacizumab and irinotecan in 72 children with recurrent LGG 


\begin{tabular}{|lll|}
\hline Toxicity & Grade & No of patients \\
\hline Vomiting & $1-2$ & $42(58 \%)$ \\
\hline Diarrhea & $1-2$ & $30(42 \%)$ \\
\hline Proteinuria & $1-2$ & $21(29 \%)$ \\
\hline Hypertension & 3 & 1 \\
\hline Epistaxis & $1-2$ & $8(11 \%)$ \\
\hline Rectal bleeding & 1 & 2 \\
\hline Fatigue & $1-2$ & $12(17 \%)$ \\
\hline Alopecia & $1-2$ & $7(10 \%)$ \\
\hline Allergy & $1-2$ & $3(4 \%)$ \\
\hline Neutropenia & 2 & 1 \\
\hline Anemia & 2 & 1 \\
\hline Thrombosis & 2 & 1 \\
\hline
\end{tabular}

\section{Bevacizumab re-treatment}

Nineteen patients $(26 \%)$ were re-treated with bevacizumab. For 3 patients, the treatment had been interrupted because of toxicity, for 6 others because of a radiological stability and for the 10 remaining patients systematically after a treatment duration of one year. Thirteen patients had no other therapy in the interval between the two bevacizumab-based treatments. Bevacizumab monotherapy was used for 3 patients, associated to irinotecan for 8 and to vinblastine for 2 patients. Despite previous exposure to bevacizumab, all patients had clinical and/or radiographic improvement or stability with re-treatment. The median duration of the second bevacizumab treatment regimen was 8 months (range, 2.6-12.1 months). Median PFS after secondary therapy with bevacizumab was 12 months (range, 4-36 months). Five patients were re-treated a third time with bevacizumab associated for 2 of them with vinblastine and clinical improvement and radiological stability were again noted. One patient was treated a third and a fourth time with bevacizumab for subsequent clinical or radiographic progression. Within few months, his vision again improved in conjunction with radiographic improvement. The PFS after each bevacizumabbased therapy were respectively 12 and 20 months. Bevacizumab related toxicities reported were hypertension in two, the two times at the first bevacizumab-based regimen line but not during retreatment, and proteinuria in eight, four of them during the two lines of treatment, three only during the first and one during the re-treatment. 


\section{Discussion}

In this expanded cohort, the largest available in the literature for this disease, we confirm that the combination of bevacizumab and irinotecan produces at least a disease stabilization in over $95 \%$ of patients who had previously failed standard chemotherapy and/or radiotherapy. These results are superior to all the therapeutic combinations tested so far in this setting. Bevacizumab-based therapy demonstrated partial radiological responses in only 15 of $72(21 \%)$ of these refractory or recurrent cases, a lower response rate than in the study from Hwang (5 out of the 14 patients) [27]. Conversely, the COG phase II reported only 2 partial radiological responses in the 35 patients studied, i.e. 5.7\% [29]. Only four tumors progressed under irinotecan / bevacizumab treatment. This result is similar to what has been observed in a previous study [29].

However, radiological responses did not necessarily predict sustained disease stability. In our study, PFS at 6 and 24 months were 91.7\% [IC 95\% 85.5-98.3] and 38.2\% [IC 95\% 28.2-51.8] respectively. In the PBTC phase II study including 35 LGG patients the 6-month and 2-year progression-free survivals were similar, with $85.4 \%(\mathrm{SE}+5.96 \%)$ and $47.8 \%(\mathrm{SE}+9.27 \%)$, respectively [29].

This association also leads to rapid clinical benefits. Almost all patients $(96 \%)$ described an improvement or a stabilization of their clinical state. The relatively short time to maximal response and noted clinical improvement highlights the advantage of utilizing this regimen in situations where there is a functional threat or a progressive neurologic/visual deterioration.

Some patients were re-treated with bevacizumab and despite previous exposure, all patients had clinical and/or radiographic improvement or stability. There did not appear to be a decrease in efficacy with repeated courses of bevacizumab, implying that the tumors did not gain resistance to this approach. This suggests that the tumor vasculature might still be responsive to bevacizumab on repeated exposure. Moreover, toxicity of the second or third chemotherapy with bevacizumab was not increased.

The evaluation of potential prognostic factors demonstrated that the age at initiation of treatment affected the response and improvement of PFS. Patients younger than 5 years had a lower PFS than older patients. This observation confirms the data from the literature obtained with standard chemotherapies. Stokland et al. demonstrated on a cohort of 639 children with low-grade gliomas that age is a strong risk factor for disease progression [11, 32].

In our study, chemoresistance or anterior chemosensitivity did not impact PFS after treatment with irinotecan-bevacizumab. De Haas et al. studied relapses after a first line of chemotherapy in 68 patients treated for an optic pathways glioma. This study showed that patients with an objective radiological response to first-line chemotherapy had a greater chance of being good responders to second-line chemotherapy [11]. The use of bevacizumab has similar efficacy in patients previously chemosensitive or not. Consequently, bevacizumab-irinotecan appear to be a good rescue regimen for patients who did not respond to the previous chemotherapy(ies). 
NF1 status did not appeared to predict a more sustained disease stability after this regimen, unlike what is usually observed with standard chemotherapy [33,34]. These patients responded not longer than nonNF1 patients despite differences in tumor vascularization on MRI [35, 36].

The study of PFS according to the pathological diagnosis seems to show a poorer PFS in patients with gangliogliomas compared to patients with pilocytic astrocytoma. This difference was not statistically significant. This trend seems to be confirmed after the molecular biology study of BRAF status with poorer PFS in patients with tumors carrying the BRAF V600E mutation, but again without statistical significance. In the literature, patients with BRAF V600E PLGG exhibited poor outcomes after chemotherapy and radiation therapies and constitutes a distinct population with poor prognosis when treated with current adjuvant therapy [11]. In the absence of significant differences, this therapeutic combination could therefore be considered as a rescue regimen, irrespective of the nature of the tumor.

The pattern of toxicities in this study is similar to that observed in adult and pediatric patients treated with bevacizumab [37, 38]. In our study, hypertension, fatigue, and proteinuria were relatively common but usually manageable with prolongation of the dosing interval. Nevertheless our understanding of longterm side effects in patients exposed to bevacizumab is evolving and both short- and long-term toxicity warrants further studies [27].

The results of this study indicate that bevacizumab-irinotecan is a useful therapeutic modality for children with recurrent LGG who have failed standard chemotherapy regimens. Disease control is obtained in most patients, irrespective of their clinical characteristics. Sustained responses are mostly observed in older children. Duration of therapy does not affect the PFS after stopping the therapy. Optimal treatment duration need to be defined further.

\section{Abbreviations}

m: months, y: years, ChemoR: chemoresistant, ChemoS: chemosensitive; MR: minor response, PR: partial response, SD: stable disease, PD: progressive disease, PA/PMA: pilocytic astrocytoma/pilomyxoid astrocytoma, GG: ganglioglioma, WT: wild type, UNDETM: undetermined

\section{Declarations}

\section{Author contribution statement:}

Drs Grill, Tauziède-Espariat, Varlet and de Marcellus designed and conceptualized the report, acquired and interpreted patient data, drafted the manuscript for intellectual content, reviewed and revised the manuscript. Drs Cuinet, Pasqualini, Robert, Beccaria, Puget, Boddaert, Figarella-Branger, De Carli, Bourdeaut, Leblond, Fouyssac, Andre, Bertozzi, Butel, Dufour and Valteau-Couanet acquired and interpreted patient data, reviewed and revised the manuscript. All authors approved the final manuscript as submitted and agree to be accountable for all aspects of the work. 
Data availability: The datasets generated during and/or analysed during the current study are available from the corresponding author on reasonable request.

\section{Compliance with ethical standards}

Conflict of interest: No funds, grants, or other support was received. The authors have no relevant financial or non-financial interests to disclose. Dr Grill has received grant support from Hoffmann La Roche for a study with bevacizumab in pediatric high-grade gliomas.

Ethics approval: Ethical approval was waived by the local Ethics Committee of Gustave Roussy (CCTIRS $n^{\circ} 14.817$ ) in view of the retrospective nature of the study and all the procedures being performed were part of the routine care.

Informed consent: Informed consent was obtained from all individual participants included in the study.

\section{References}

1. Ostrom QT, Gittleman H, Fulop J et al (2015) CBTRUS Statistical Report: Primary Brain and Central Nervous System Tumors Diagnosed in the United States in 2008-2012. Neuro-Oncol 17 Suppl 4iv1iv62. https://doi.org/10.1093/neuonc/nov189

2. Gajjar A, Sanford RA, Heideman R et al (1997) Low-grade astrocytoma: a decade of experience at St. Jude Children's Research Hospital. J Clin Oncol Off J Am Soc Clin Oncol 15:2792-2799

3. Bowers DC, Gargan L, Kapur P et al (2003) Study of the MIB-1 labeling index as a predictor of tumor progression in pilocytic astrocytomas in children and adolescents. J Clin Oncol Off J Am Soc Clin Oncol 21:2968-2973. https://doi.org/10.1200/JC0.2003.01.017

4. Merchant TE, Kun LE, Wu S et al (2009) Phase II trial of conformal radiation therapy for pediatric lowgrade glioma. J Clin Oncol Off J Am Soc Clin Oncol 27:3598-3604. https://doi.org/10.1200/JCO.2008.20.9494

5. Massimino M, Spreafico F, Cefalo $\mathrm{G}$ et al (2002) High response rate to cisplatin/etoposide regimen in childhood low-grade glioma. J Clin Oncol Off J Am Soc Clin Oncol 20:4209-4216

6. Gururangan S, Cavazos CM, Ashley D et al (2002) Phase II study of carboplatin in children with progressive low-grade gliomas. J Clin Oncol Off J Am Soc Clin Oncol 20:2951-2958

7. Bouffet $E$, Jakacki R, Goldman S et al (2012) Phase II study of weekly vinblastine in recurrent or refractory pediatric low-grade glioma. J Clin Oncol Off J Am Soc Clin Oncol 30:1358-1363. https://doi.org/10.1200/JC0.2011.34.5843

8. Laithier V, Grill J, Le Deley M-C et al (2003) Progression-free survival in children with optic pathway tumors: dependence on age and the quality of the response to chemotherapy-results of the first French prospective study for the French Society of Pediatric Oncology. J Clin Oncol Off J Am Soc Clin Oncol 21:4572-4578. https://doi.org/10.1200/JC0.2003.03.043 
9. Ater JL, Zhou T, Holmes E et al (2012) Randomized Study of Two Chemotherapy Regimens for Treatment of Low-Grade Glioma in Young Children: A Report From the Children's Oncology Group. J Clin Oncol 30:2641-2647. https://doi.org/10.1200/JC0.2011.36.6054

10. Gnekow AK, Walker DA, Kandels D et al (2017) A European randomised controlled trial of the addition of etoposide to standard vincristine and carboplatin induction as part of an 18-month treatment programme for childhood ( $\leq 16$ years) low grade glioma - A final report. Eur J Cancer Oxf Engl 1990 81:206-225. https://doi.org/10.1016/j.ejca.2017.04.019

11. de Haas V, Grill J, Raquin M-A et al (2009) Relapses of optic pathway tumors after first-line chemotherapy. Pediatr Blood Cancer 52:575-580. https://doi.org/10.1002/pbc.21911

12. Bartels U, Hawkins C, Jing M et al (2006) Vascularity and angiogenesis as predictors of growth in optic pathway/hypothalamic gliomas. J Neurosurg 104:314-320. https://doi.org/10.3171/ped.2006.104.5.314

13. Ferrara N, Gerber H-P, LeCouter J (2003) The biology of VEGF and its receptors. Nat Med 9:669-676. https://doi.org/10.1038/nm0603-669

14. Jain RK (2008) Lessons from multidisciplinary translational trials on anti-angiogenic therapy of cancer. Nat Rev Cancer 8:309-316. https://doi.org/10.1038/nrc2346

15. Gremonprez F, Descamps B, Izmer A et al (2015) Pretreatment with VEGF(R)-inhibitors reduces interstitial fluid pressure, increases intraperitoneal chemotherapy drug penetration, and impedes tumor growth in a mouse colorectal carcinomatosis model. Oncotarget 6:29889-29900. https://doi.org/10.18632/oncotarget.5092

16. Friedman HS, Petros WP, Friedman AH et al (1999) Irinotecan therapy in adults with recurrent or progressive malignant glioma. J Clin Oncol Off J Am Soc Clin Oncol 17:1516-1525

17. Vredenburgh JJ, Desjardins A, Reardon DA, Friedman HS (2009) Experience with irinotecan for the treatment of malignant glioma. Neuro-Oncol 11:80-91. https://doi.org/10.1215/15228517-2008-075

18. Sapra P, Kraft P, Pastorino F et al (2011) Potent and sustained inhibition of HIF-1 a and downstream genes by a polyethyleneglycol-SN38 conjugate, EZN-2208, results in anti-angiogenic effects. Angiogenesis 14:245-253. https://doi.org/10.1007/s10456-011-9209-1

19. Hurwitz H, Fehrenbacher L, Novotny W et al (2004) Bevacizumab plus irinotecan, fluorouracil, and leucovorin for metastatic colorectal cancer. N Engl J Med 350:2335-2342. https://doi.org/10.1056/NEJMoa032691

20. Sandler A, Gray R, Perry MC et al (2006) Paclitaxel-carboplatin alone or with bevacizumab for nonsmall-cell lung cancer. N Engl J Med 355:2542-2550. https://doi.org/10.1056/NEJMoa061884

21. Yang JC, Haworth L, Sherry RM et al (2003) A randomized trial of bevacizumab, an anti-vascular endothelial growth factor antibody, for metastatic renal cancer. N Engl J Med 349:427-434. https://doi.org/10.1056/NEJMoa021491

22. Perren TJ, Swart AM, Pfisterer J et al (2011) A phase 3 trial of bevacizumab in ovarian cancer. N Engl J Med 365:2484-2496. https://doi.org/10.1056/NEJMoa1103799 
23. Chisholm JC, Merks JHM, Casanova M et al (2017) Open-label, multicentre, randomised, phase II study of the EpSSG and the ITCC evaluating the addition of bevacizumab to chemotherapy in childhood and adolescent patients with metastatic soft tissue sarcoma (the BERNIE study). Eur $\mathrm{J}$ Cancer Oxf Engl 1990 83:177-184. https://doi.org/10.1016/j.ejca.2017.06.015

24. Grill J, Massimino M, Bouffet E et al (2018) Phase Il, Open-Label, Randomized, Multicenter Trial (HERBY) of Bevacizumab in Pediatric Patients With Newly Diagnosed High-Grade Glioma. J Clin Oncol Off J Am Soc Clin Oncol 36:951-958. https://doi.org/10.1200/JC0.2017.76.0611

25. Packer RJ, Jakacki R, Horn M et al (2009) Objective response of multiply recurrent low-grade gliomas to bevacizumab and irinotecan. Pediatr Blood Cancer 52:791-795. https://doi.org/10.1002/pbc. 21935

26. Couec M-L, André N, Thebaud E et al (2012) Bevacizumab and irinotecan in children with recurrent or refractory brain tumors: toxicity and efficacy trends. Pediatr Blood Cancer 59:34-38. https://doi.org/10.1002/pbc.24066

27. Hwang El, Jakacki RI, Fisher MJ et al (2013) Long-term efficacy and toxicity of bevacizumab-based therapy in children with recurrent low-grade gliomas. Pediatr Blood Cancer 60:776-782. https://doi.org/10.1002/pbc.24297

28. Sikkema AH, de Bont ESJM, Molema G et al (2011) Vascular endothelial growth factor receptor 2 (VEGFR-2) signalling activity in paediatric pilocytic astrocytoma is restricted to tumour endothelial cells. Neuropathol Appl Neurobiol 37:538-548. https://doi.org/10.1111/j.1365-2990.2011.01160.x

29. Gururangan S, Fangusaro J, Poussaint TY et al (2014) Efficacy of bevacizumab plus irinotecan in children with recurrent low-grade gliomas-a Pediatric Brain Tumor Consortium study. Neuro-Oncol 16:310-317. https://doi.org/10.1093/neuonc/not154

30. Gururangan S, Chi SN, Young Poussaint T et al (2010) Lack of efficacy of bevacizumab plus irinotecan in children with recurrent malignant glioma and diffuse brainstem glioma: a Pediatric Brain Tumor Consortium study. J Clin Oncol Off J Am Soc Clin Oncol 28:3069-3075. https://doi.org/10.1200/JC0.2009.26.8789

31. Fangusaro J, Witt O, Hernáiz Driever P et al (2020) Response assessment in paediatric low-grade glioma: recommendations from the Response Assessment in Pediatric Neuro-Oncology (RAPNO) working group. Lancet Oncol 21:e305-e316. https://doi.org/10.1016/S1470-2045(20)30064-4

32. Stokland T, Liu J-F, Ironside JW et al (2010) A multivariate analysis of factors determining tumor progression in childhood low-grade glioma: a population-based cohort study (CCLG CNS9702). Neuro-Oncol 12:1257-1268. https://doi.org/10.1093/neuonc/noq092

33. Lassaletta A, Scheinemann K, Zelcer SM et al (2016) Phase II Weekly Vinblastine for ChemotherapyNaïve Children With Progressive Low-Grade Glioma: A Canadian Pediatric Brain Tumor Consortium Study. J Clin Oncol Off J Am Soc Clin Oncol 34:3537-3543. https://doi.org/10.1200/JC0.2016.68.1585

34. Ater JL, Xia C, Mazewski CM et al (2016) Nonrandomized comparison of neurofibromatosis type 1 and non-neurofibromatosis type 1 children who received carboplatin and vincristine for progressive 
low-grade glioma: A report from the Children's Oncology Group. Cancer 122:1928-1936.

https://doi.org/10.1002/cncr.29987

35. Jost SC, Ackerman JW, Garbow JR et al (2008) Diffusion-weighted and dynamic contrast-enhanced imaging as markers of clinical behavior in children with optic pathway glioma. Pediatr Radiol 38:1293-1299. https://doi.org/10.1007/s00247-008-1003-x

36. Maloney E, Stanescu AL, Perez FA et al (2018) Surveillance magnetic resonance imaging for isolated optic pathway gliomas: is gadolinium necessary? Pediatr Radiol 48:1472-1484. https://doi.org/10.1007/s00247-018-4154-4

37. Gressett SM, Shah SR (2009) Intricacies of bevacizumab-induced toxicities and their management. Ann Pharmacother 43:490-501. https://doi.org/10.1345/aph.1L426

38. Glade Bender JL, Adamson PC, Reid JM et al (2008) Phase I trial and pharmacokinetic study of bevacizumab in pediatric patients with refractory solid tumors: a Children's Oncology Group Study. J Clin Oncol Off J Am Soc Clin Oncol 26:399-405. https://doi.org/10.1200/JC0.2007.11.9230

\section{Figures}

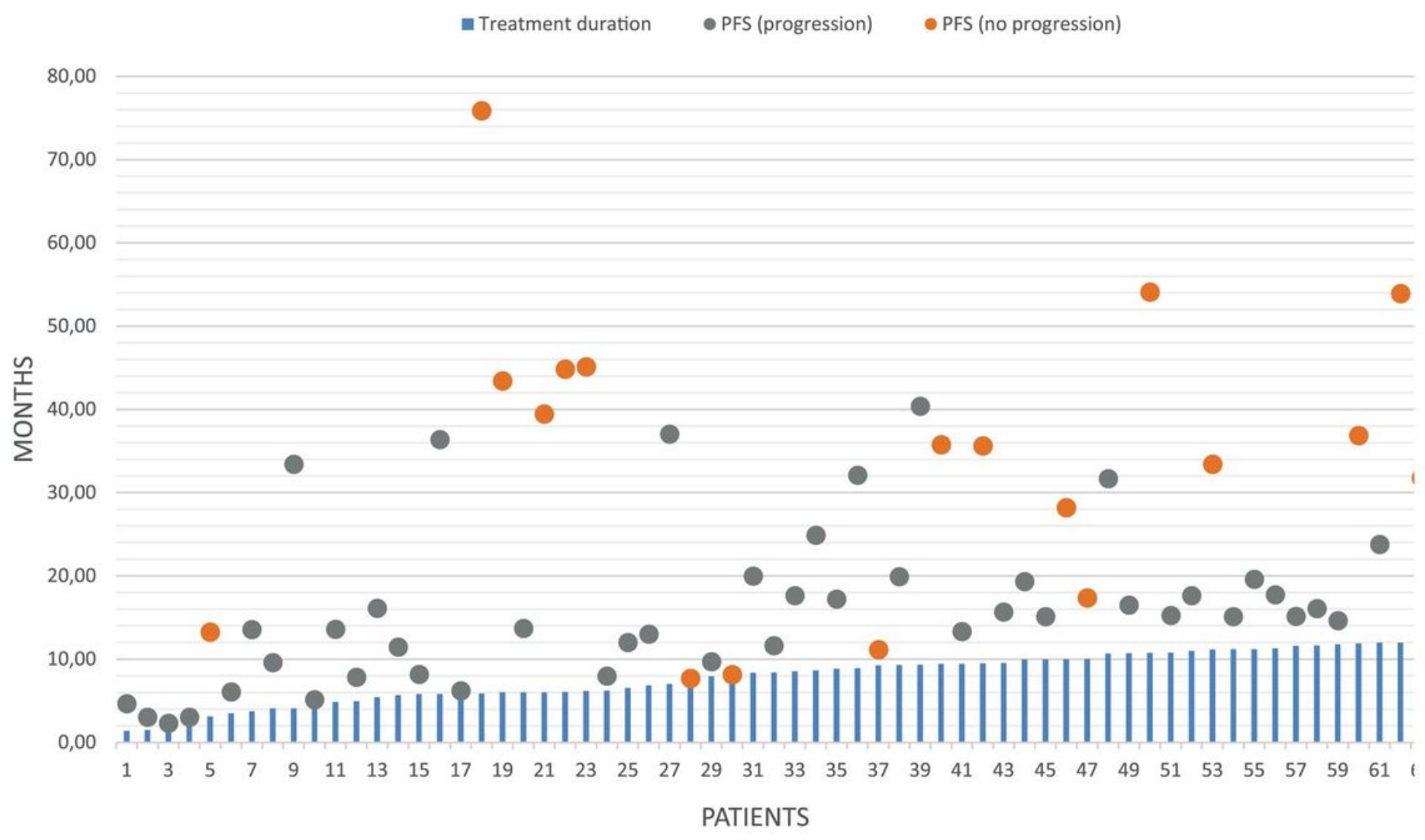

Figure 1 
Treatment duration (blue histogram) , PFS and time to last follow-up in 72 patients treated with irinotecan and bevacizumab are shown as grey dots in case of recurrence or as orange dots in case of ongoing progression-free survival.

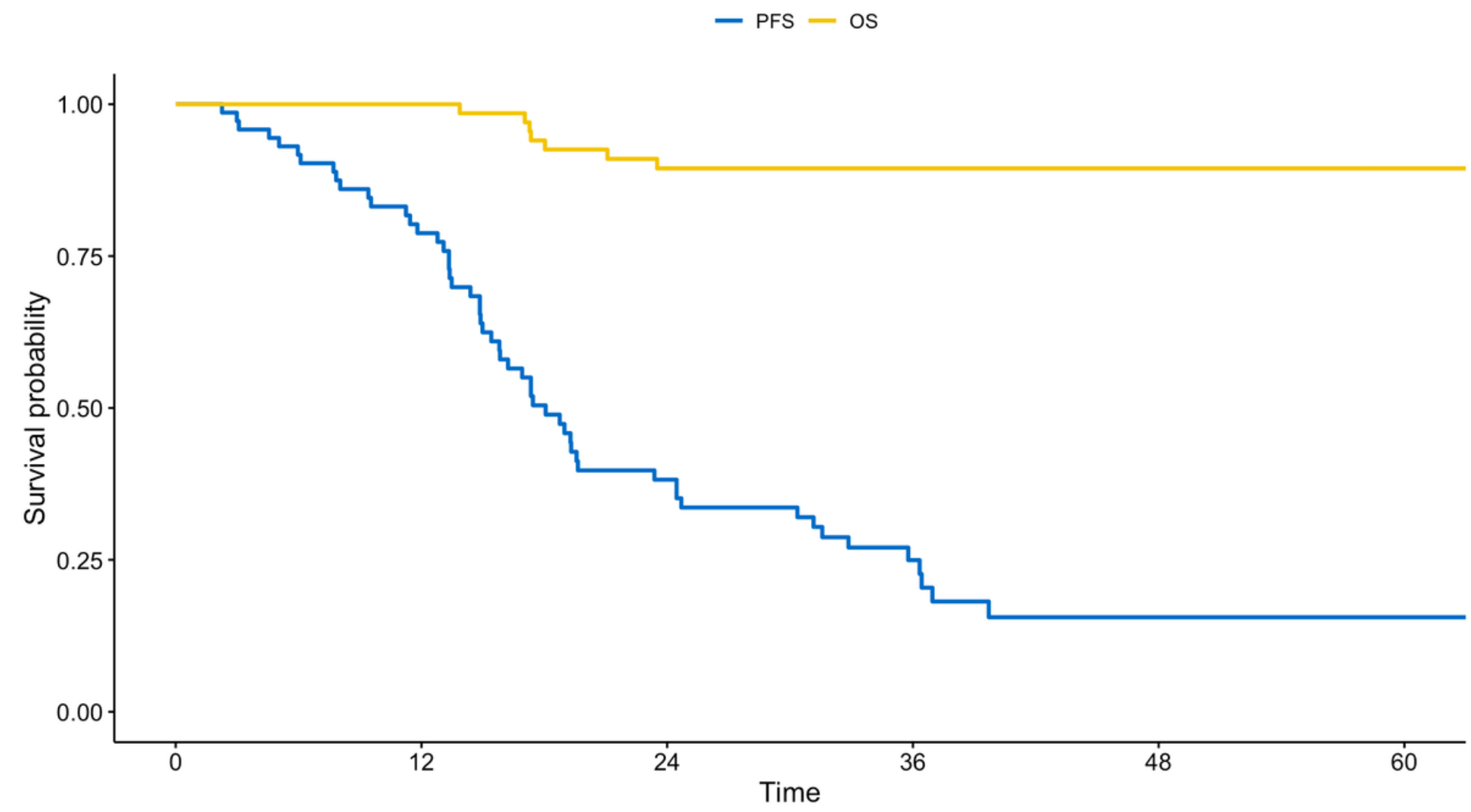

Number at risk

\begin{tabular}{|c|c|c|c|c|c|}
\hline PFS $\quad 72$ & 54 & 25 & 12 & 3 & 1 \\
\hline 72 & 68 & 57 & 48 & 28 & 21 \\
\hline
\end{tabular}

\section{Figure 2}

Overall survival (OS) and progression-free survival (PFS) in 72 patients treated with irinotecan and bevacizumab. 

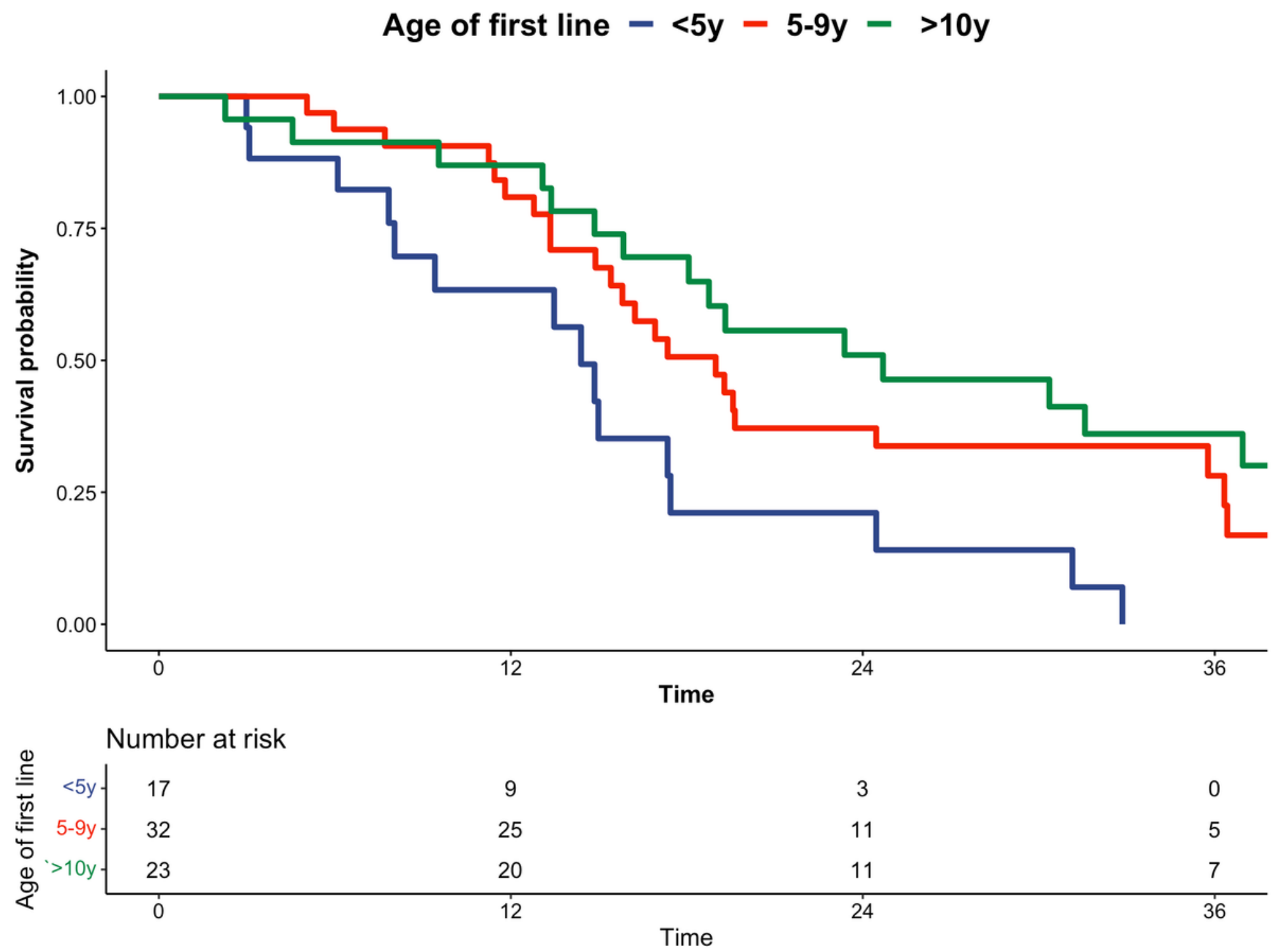

Figure 3

PFS according to age at initiation of initial treatment. 


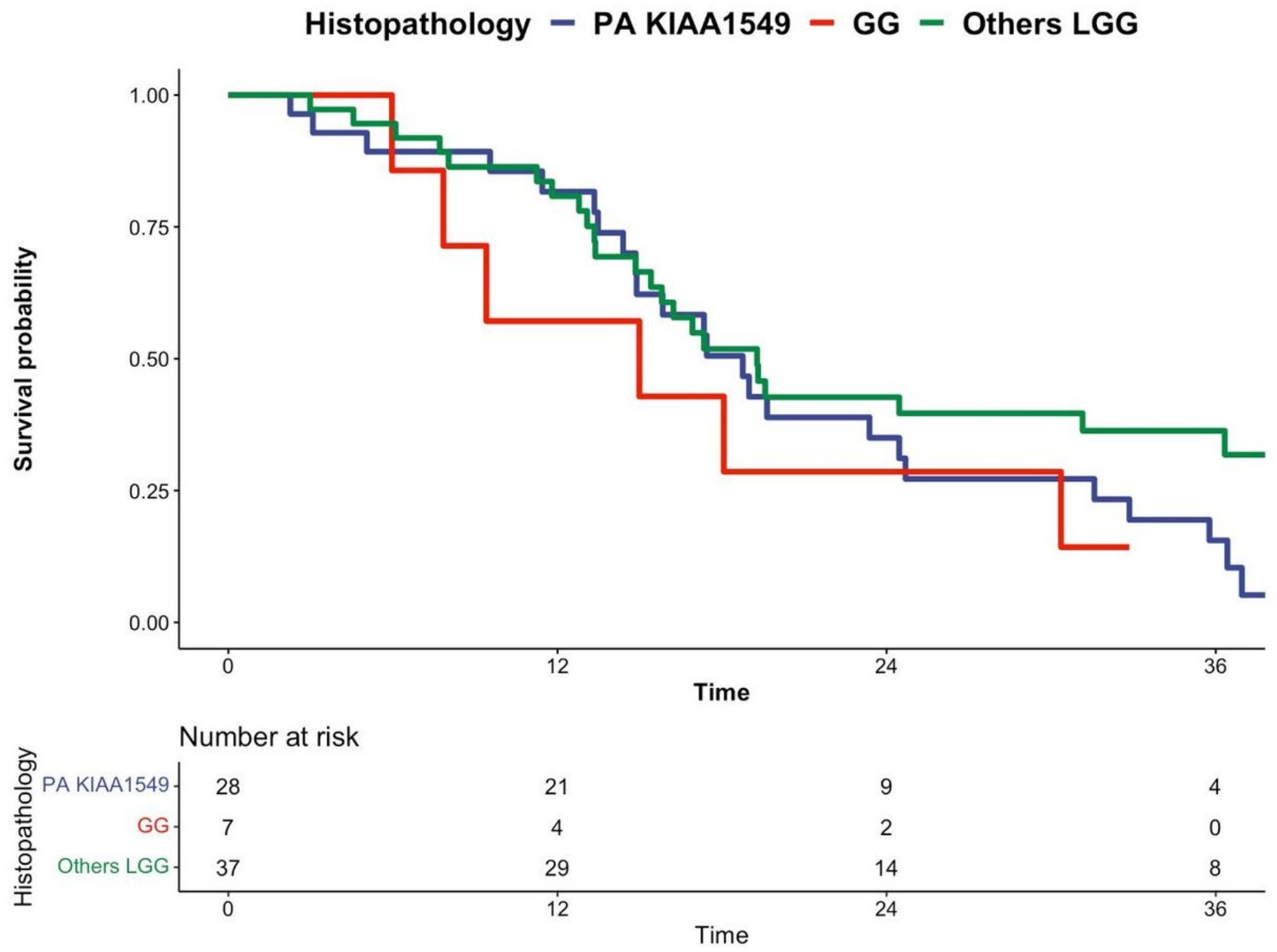

Figure 4

PFS according to histopathology. (GG: Ganglioglioma, LGG: low grade glioma) 


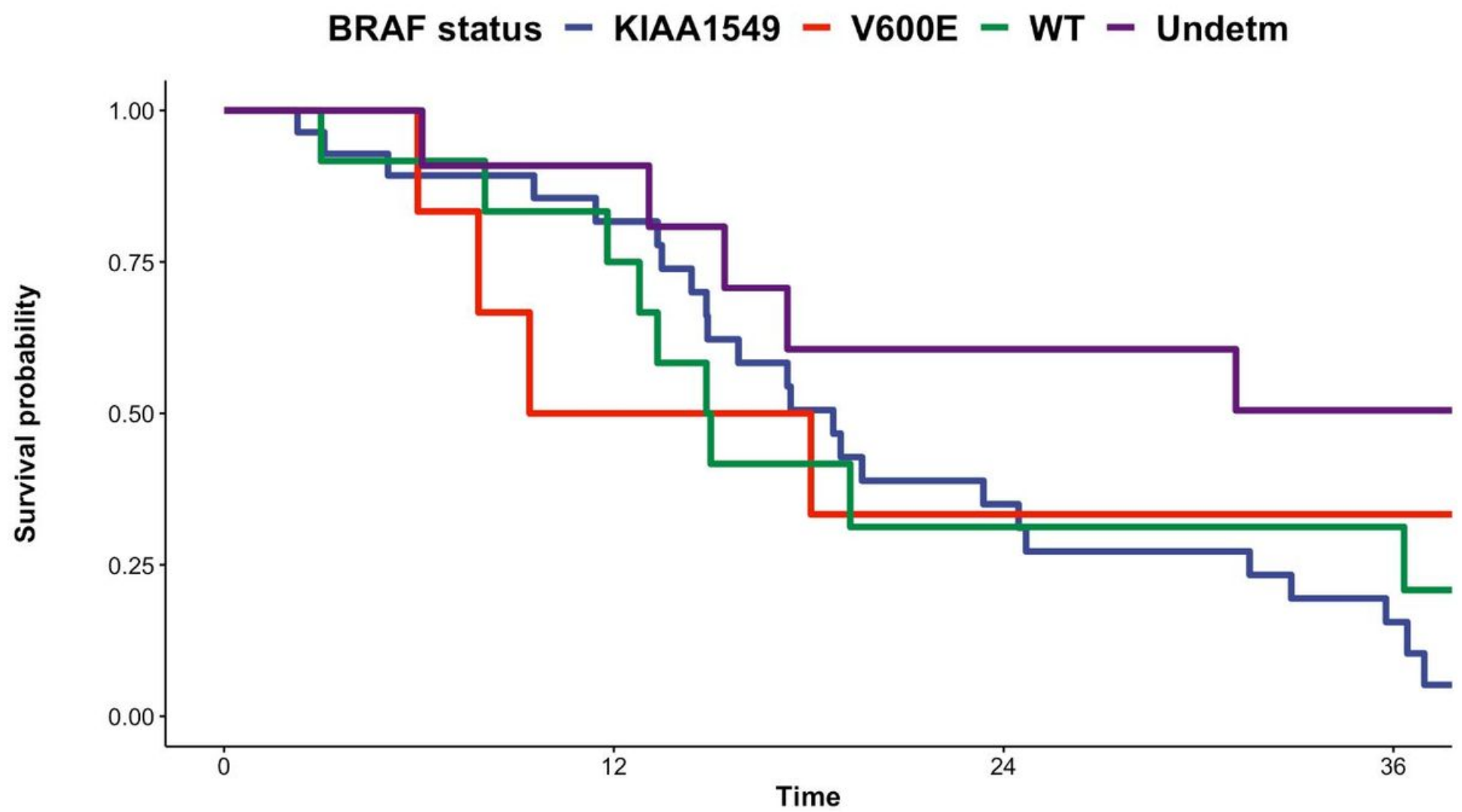

\begin{tabular}{|c|c|c|c|c|}
\hline \multirow{2}{*}{$\stackrel{\infty}{己}$ KIAA1549 } & \multicolumn{4}{|c|}{ Number at risk } \\
\hline & 28 & 21 & 9 & 4 \\
\hline$\frac{\pi}{\omega} \quad V 600 E$. & 6 & 3 & 2 & 1 \\
\hline WT. & 12 & 9 & 3 & 3 \\
\hline Undetm. & 11 & 9 & 6 & 3 \\
\hline & 0 & 12 & 24 & 36 \\
\hline
\end{tabular}

Figure 5

PFS according to BRAF status. (WT: wild type, Undetm: undetermined) 

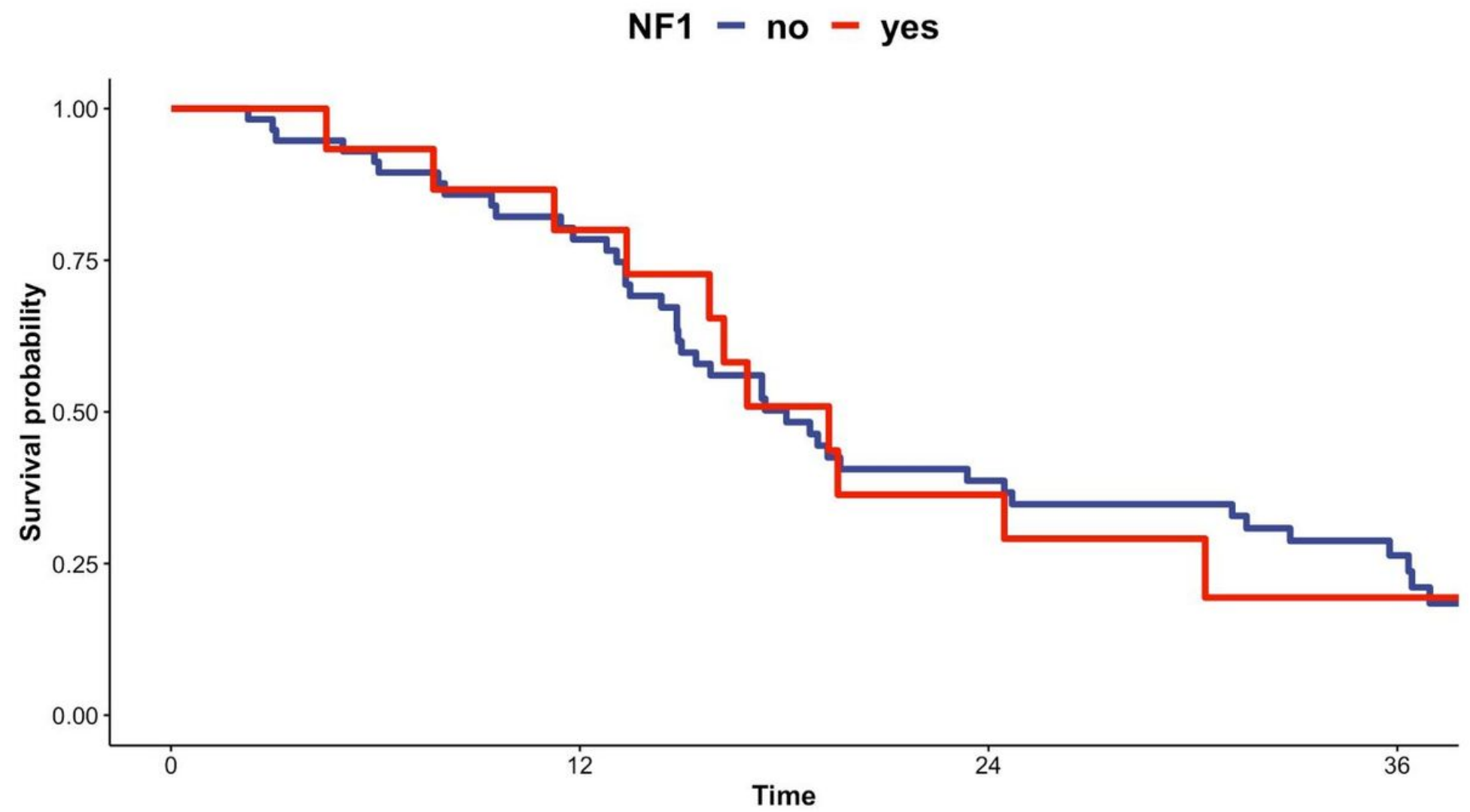

Number at risk

$\underset{\mathbf{z}}{\mathbf{z}}$ yes \begin{tabular}{ccccc}
57 & 42 & 20 & 11 \\
15 & 12 & 5 & 1 \\
\hline 0 & 12 & Time & 24 & 36
\end{tabular}

Figure 6

PFS according to NF1 status. 


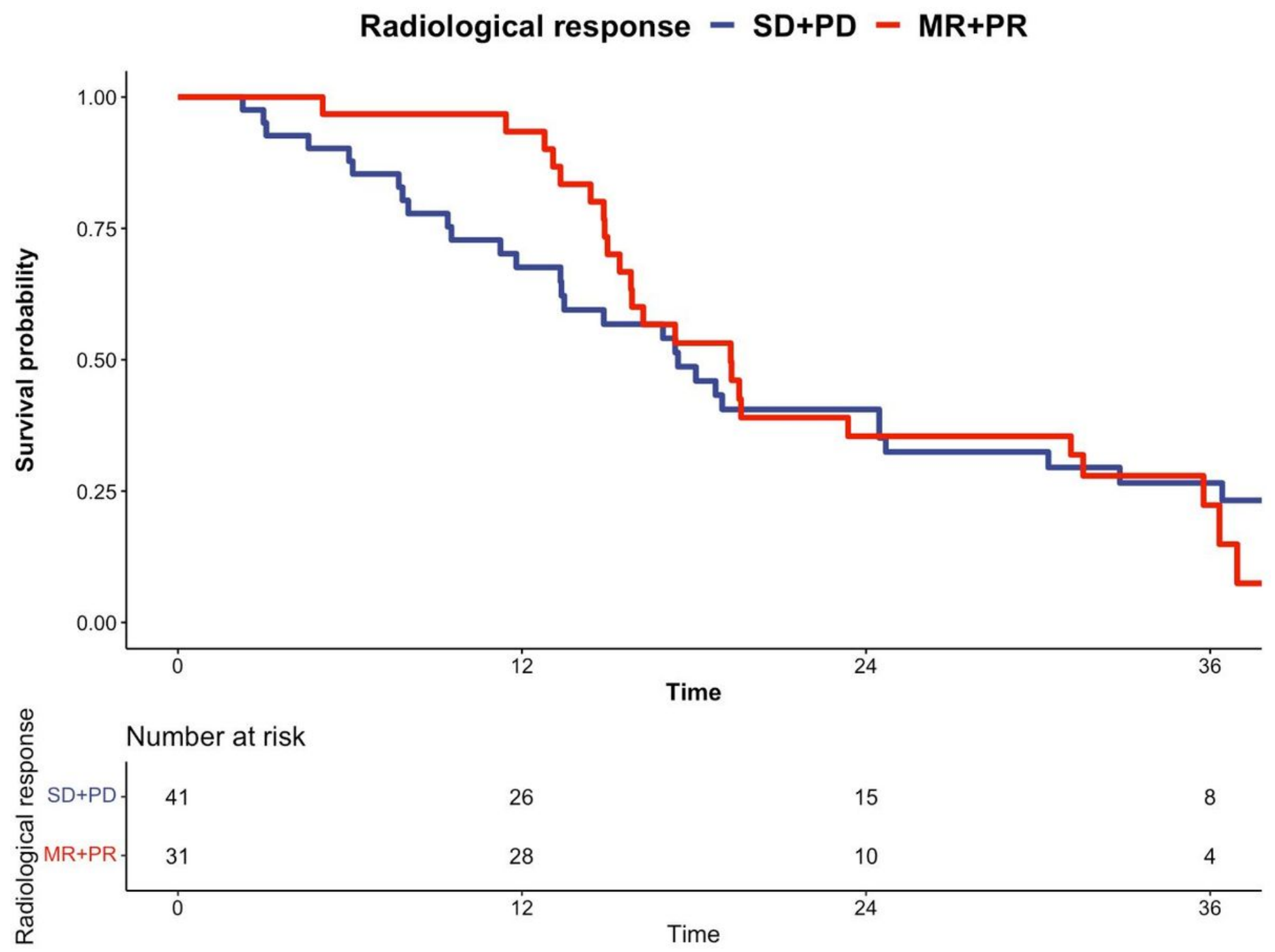

Figure 7

PFS according to radiological response. (MR: minor response, PR: partial response, SD: stable disease, PD: progressive disease) 


\section{Treatment duration $-<9$ months $->9$ months}

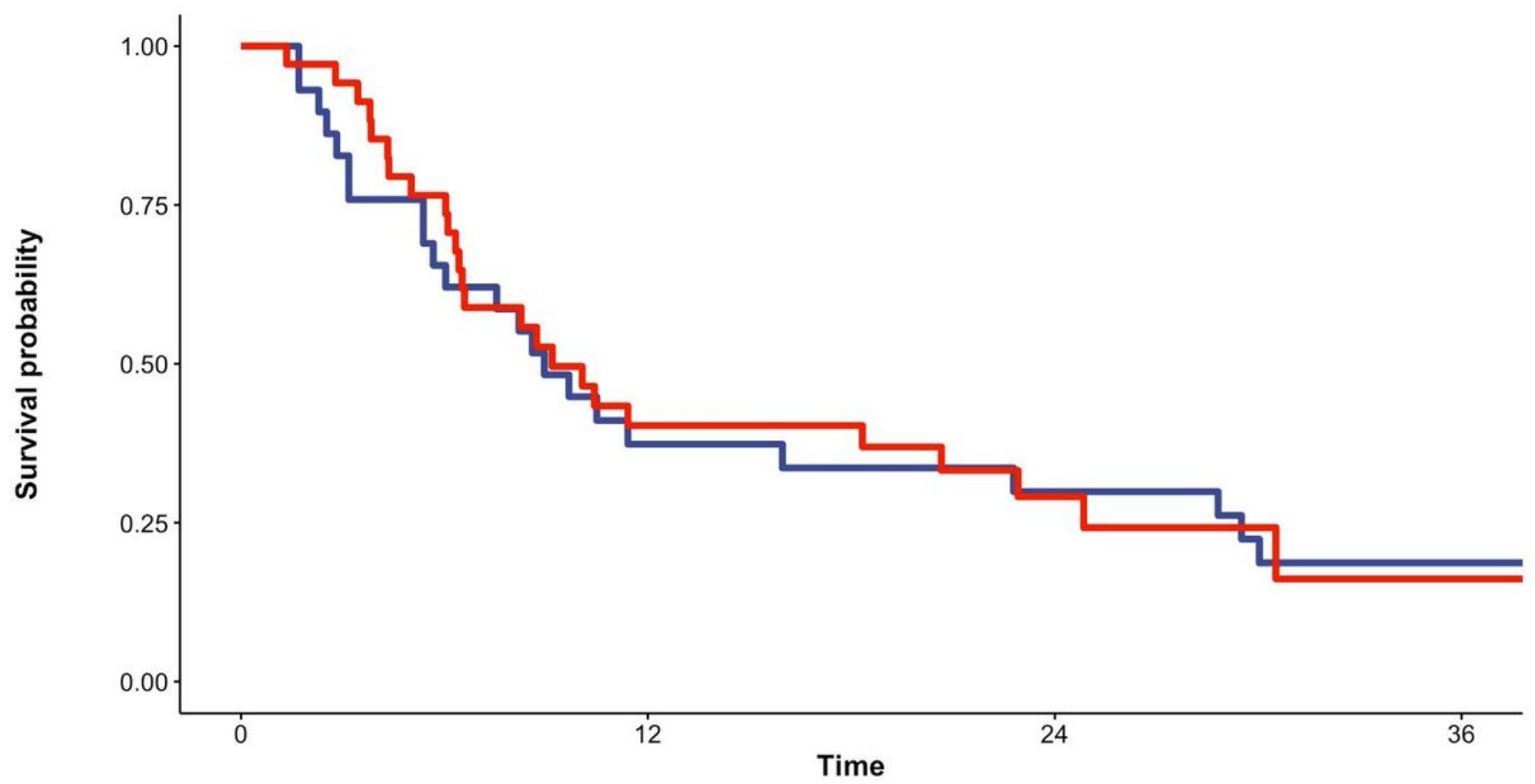

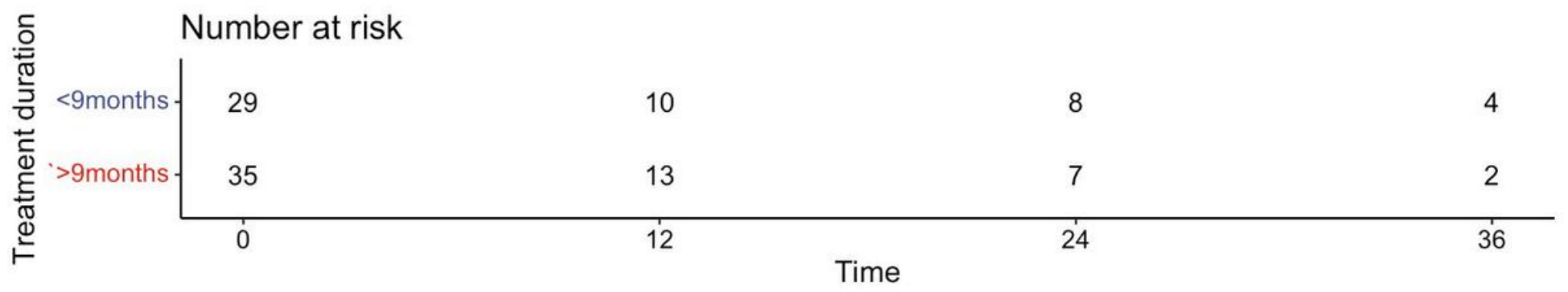

Figure 8

PFS according to treatment duration. 


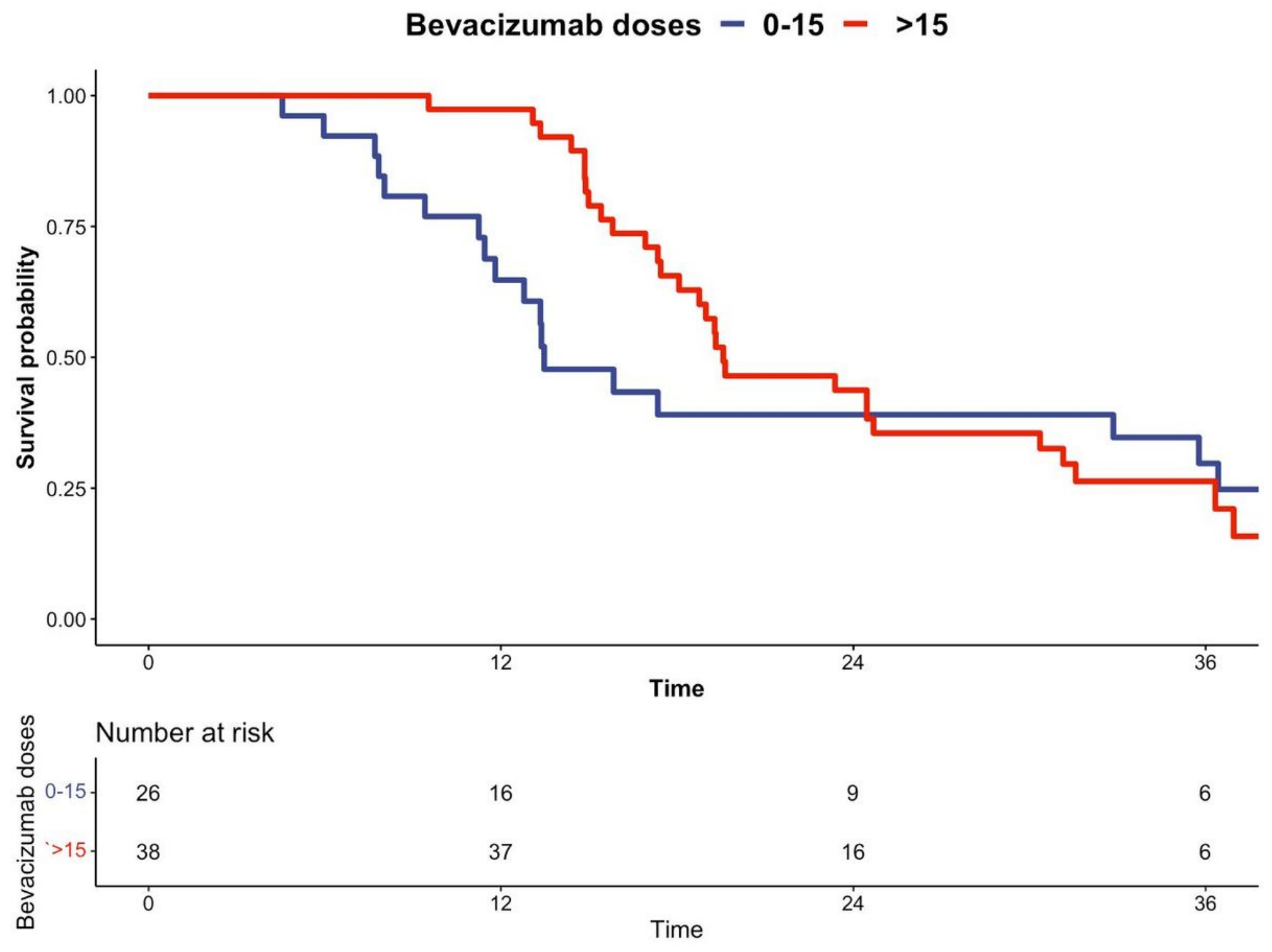

Figure 9

PFS according to the number of bevacizumab doses. 


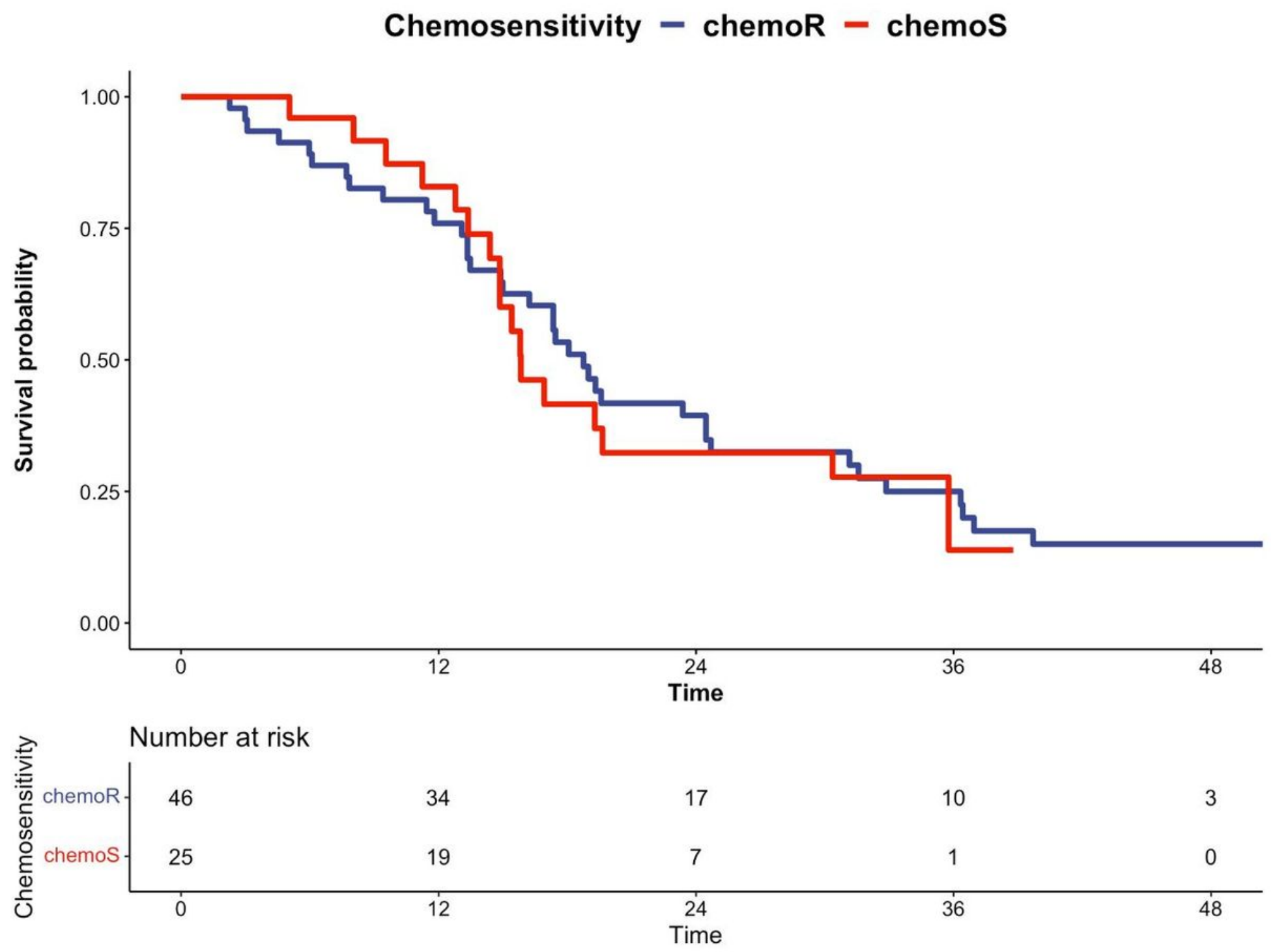

Figure 10

PFS according to prior chemosensitivity. (chemoR: chemoresistant, chemoS: chemosensitive) 